Article

\title{
Analysis of SARS-CoV-2 Screening Clinic (Including Drive-through System) Data at a Single University Hospital in South Korea from 27 January 2020 to 31 March 2020 During the COVID-19 Outbreak
}

\author{
Min Cheol Chang ${ }^{1}$, Wan-Seok Seo ${ }^{2}$, Donghwi Park ${ }^{3, *}$ and Jian Hur ${ }^{4, *}$ \\ 1 Department of Physical Medicine and Rehabilitation, College of Medicine, Yeungnam University, \\ Daegu 42415, Korea; wheel633@ynu.ac.kr \\ 2 Department of Psychiatry, College of Medicine, Yeungnam University, Daegu 42415, Korea; \\ sws3901@ynu.ac.kr \\ 3 Department of Physical Medicine and Rehabilitation, Ulsan University Hospital, \\ University of Ulsan College of Medicine, Ulsan 44033, Korea \\ 4 Department of Infectious Disease Internal Medicine, College of Medicine, Yeungnam University, \\ Daegu 42415, Korea \\ * Correspondence: bdome@hanmail.net (D.P.); sarang7529@hanmail.net (J.H.)
}

Received: 28 April 2020; Accepted: 22 May 2020; Published: 26 May 2020

\begin{abstract}
In this study, we evaluated the efficiency of a drive-through (DT) screening system for severe acute respiratory syndrome coronavirus 2 (SARS-CoV-2) by comparing it with a conventional screening system. We reviewed and analyzed the SARS-CoV-2 screening data obtained at our university hospital. We compared the number of tests for SARS-CoV-2 (using real-time polymerase chain reaction) performed using two different specimen collection systems-DT and conventional-during the coronavirus disease 2019 (COVID-19) outbreak in Daegu. Based on the results, the DT screening system collected 5.8 times more specimens for testing than the conventional screening system. From 27 January to 31 March 2020, 6211 individuals were screened for SARS-CoV-2 infection using either the DT or conventional system. In total, 217 individuals tested positive for SARS-CoV-2 (positive rate: $3.50 \%$ ). Of the 6211 individuals, 3368 were symptomatic or had a history of contact with COVID-19 patients, and 142 of them tested positive for SARS-CoV-2 (positive rate: $4.22 \%$ ). Further, 2843 individuals were asymptomatic and had no history of contact with COVID-19 patients, and 75 of them tested positive for SARS-CoV-2 (positive rate: $2.64 \%$ ). In conclusion, the DT system allowed clinicians to collect specimens for SARS-CoV-2 screening more efficiently than the conventional system. Furthermore, as there might be several COVID-19 patients who remain asymptomatic, expanding the screening test to asymptomatic individuals would be necessary.
\end{abstract}

Keywords: COVID-19; drive-through screening system; real-time polymerase chain reaction

\section{Introduction}

After the first reported case of coronavirus disease 2019 (COVID-19) in Hubei Province of China in December 2019, COVID-19 cases have been reported in most countries worldwide. The novel severe acute respiratory syndrome coronavirus 2 (SARS-CoV-2, previously known as 2019-nCoV) has been rapidly spreading, with unprecedented propagation, because of its highly infectious nature. The World Health Organization declared the COVID-19 outbreak a pandemic on 11 March 2020 [1-3]. The fatality rate of COVID-19 is $2-6 \%$ and is much higher in older populations and those with underlying diseases [1-3]. The number of suspected or symptomatic individuals with COVID-19 is 
continuously increasing; hence, screening clinics, separated from other existing clinics, are being set up in several hospitals in each community.

In Daegu, South Korea, a rapid surge in COVID-19 cases occurred in late February and early March 2020. Over 5000 confirmed cases of COVID-19 were reported during this period in Daegu. For efficient and safe screening, some hospitals in Daegu implemented a drive-through (DT) screening system [4]. The individuals who used this system could provide a sample for SARS-CoV-2 testing without leaving their cars. We believe that this system is helpful for conducting rapid and safe testing during the current COVID-19 outbreak in Daegu.

In the current study, we evaluated the efficiency of a DT screening system for SARS-CoV-2 by comparing it with a conventional screening system. Moreover, we reviewed the results of SARS-CoV-2 screening (for samples collected via DT and conventional systems) in a single university hospital during the COVID-19 outbreak in Daegu, South Korea.

\section{Methods}

This study was approved by the Institutional Review Board (IRB) of Yeungnam University Hospital, and the requirement for informed consent was waived by the Ethics Committee. We reviewed and analyzed the data of a SARS-CoV-2 screening clinic at Yeungnam University Hospital, Daegu, which is one of the four university hospitals in Daegu. The data were collected using a computerized system. At the time of patient examination, the staff input the data, such as the presence of COVID-19 symptoms and a history of contact with COVID-19 patients, into the hospital's computerized system. In addition, we described the details of the DT screening system and the conventional screening system and compared the two systems. This research was approved by IRB at Yeungnam University Hospital (2020-03-101).

\section{Results}

\subsection{Operational Differences between the Conventional and Drive-Through Systems}

\subsubsection{Conventional System}

The conventional specimen collection system used a negative-pressure tent. The medical staff working in the temporary buildings wore personal protective equipment, including inner and outer gloves; an N95 respirator; an eye shield, a face shield, or goggles; a hooded coverall or gown. The outer gloves and disposable plastic apron (AP) gowns were changed after contact with every patient. The tent was divided into three sections-the section through which the individual to be tested entered (Section A), the section where the medical staff completed the questionnaires (Section B), and the section where the specimen for testing was collected (Section C) (Figure 1). When a testee entered the negative-pressure tent (Section A), the medical staff in Section B interviewed the testee. These two sections ( $A$ and $B$ ) were separated by transparent plastic. After completing the questionnaire, the testee moved to Section C, where the test specimens were collected. In Section C, three or four medical staff were waiting-one was responsible for collecting the payment for the test, one was responsible for specimen collection, and the others were responsible for sterilizing the space after the individual exited the tent. To prevent cross-contamination by testees, all sections, except Section B, were sterilized for at least 30 minutes before the next testee entered. 


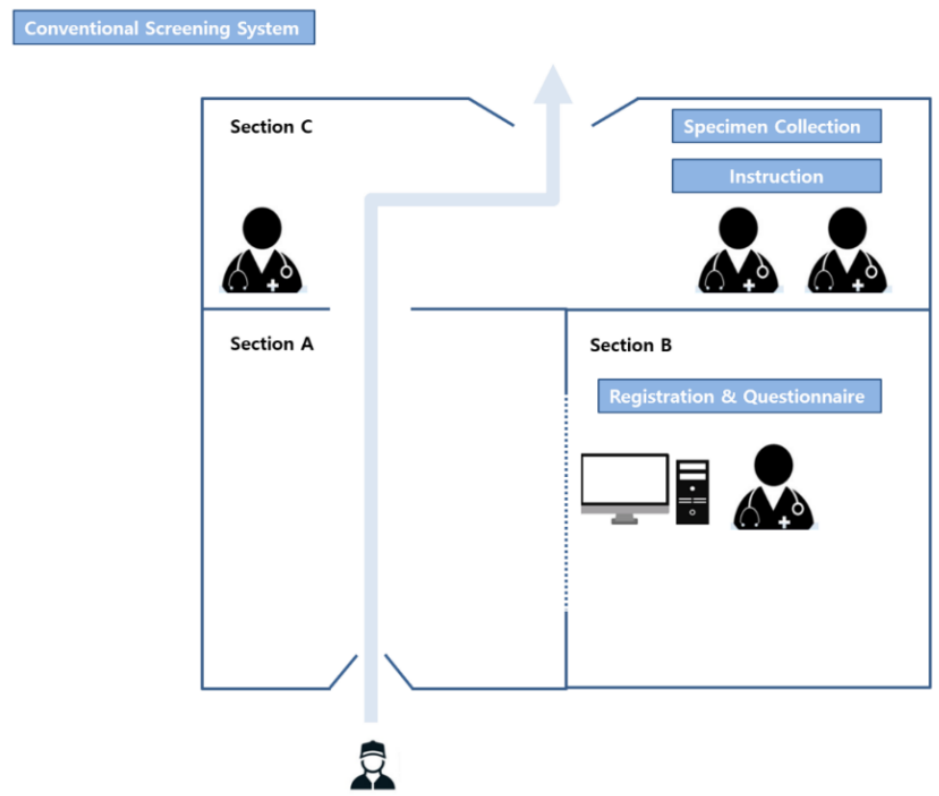

Figure 1. Illustration of the conventional severe acute respiratory syndrome coronavirus 2 (SARS-CoV-2) screening process.

\subsubsection{Drive-Through System}

The procedure for the DT specimen collection system was as follows: entrance, registration and questionnaire completion, examination, specimen collection, and receipt of instructions and information (Figure 2) [4]. For registration and questionnaire completion, examination, specimen collection, and receipt of instructions and the information leaflet, one temporary outside building was built for each section; therefore, four temporary buildings were built in total. One or two medical staff were present in each section and were completely isolated from the outdoor environment. The medical staff working in the temporary buildings wore personal protective equipment, including inner and outer gloves; an N95 respirator; an eye shield, a face shield, or goggles; a hooded coverall or gown. The outer gloves and disposable plastic apron (AP) gowns were changed after contact with every patient. In the registration and questionnaire section, the identification card of the testee was scanned and automatically transferred to the computerized system of the hospital to avoid direct contact with testees. In the registration and questionnaire and examination sections, microphones were installed inside and outside of the temporary buildings so that the medical staff could communicate with the testee without direct contact. In the specimen collection section, the driver opened the window of the car and collected his or her own specimen. In the instructions and information section, the medical staff provided information regarding when the results would be available through a microphone. The entire service could be provided to individuals without leaving their cars. Unlike conventional systems, the DT system did not require sterilization of spaces between testees, thus markedly reducing the preparation times. The total time spent on each testee was 5-7 min. Comparing the time taken for the COVID-19 test between the DT system (5-7 $\mathrm{min}$ ) and conventional system (30 min), the DT system was 4-6 times more efficient than the conventional screening system.

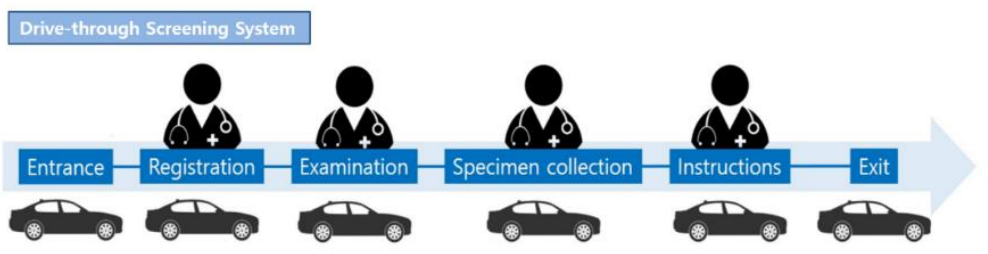

Figure 2. Illustration of the drive-through SARS-CoV-2 screening process. 


\subsection{The Difference in Specimen Collection Ability between Drive-Through and Conventional Systems}

We compared the number of specimens collected by the two different systems over a 14-day period (26 February 2020 to 10 March 2020), during which both systems were operating simultaneously. Through this, we aimed to indirectly compare the efficiency of the two systems. In the DT and conventional systems, the number of specimens collected per day were 241.7 and 41.4, respectively (3384 (DT system) vs. 580 (conventional system)). According to the total number of tests performed during the 14-day period, the efficiency of the DT system was 5.8 times higher than that of the conventional screening system. In addition, per test, the DT system was 4-6 times faster than the conventional system (5-7 min vs. $30 \mathrm{~min}$ ). Because of the higher efficiency of the DT system, as of 11 March 2020, we stopped using the conventional system and used only the DT system to collect specimens for SARS-CoV-2 testing.

\subsection{Laboratory Data}

Real-time polymerase chain reaction (Allplex ${ }^{\mathrm{TM}}$ 2019-nCoV Assay, Seegene ${ }^{\circledR}$, Seoul, Korea) was used for SARS-CoV-2 testing. From 17 January to mid-February 2020, this test was performed in less than 10 individuals per day. The first confirmed case of COVID-19 was reported on 18 February 2020, and a gradual increase in the number of tests and confirmed cases followed. After the sudden outbreak of COVID-19 in Daegu, the number of tests, newly confirmed cases, and positive rate sharply increased. In mid-March 2020, 150-450 individuals were being tested for SARS-CoV-2 daily (Figure 3 and Table 1).

(A)

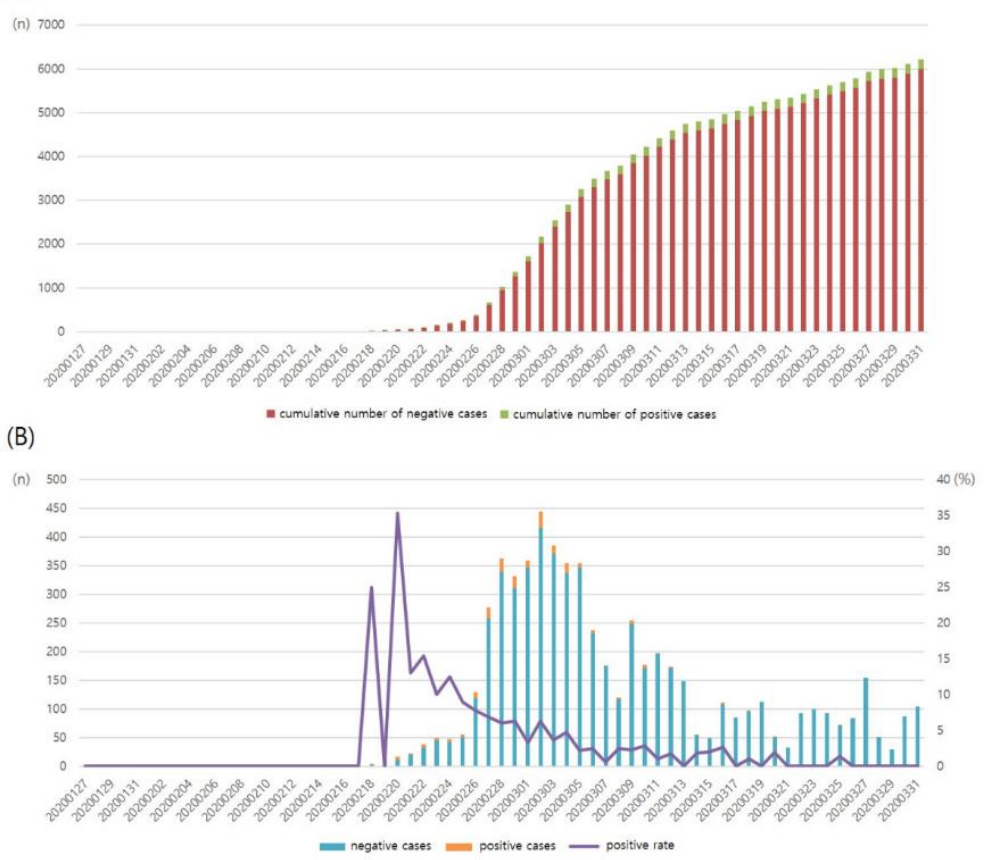

Figure 3. (A) Graph showing the cumulative number of positive and negative cases after SARS-CoV-2 testing and positive test result rate by date in the screening clinic of our university hospital. (B) Graph showing the daily number of positive and negative cases after SARS-CoV-2 testing. 
Table 1. A cumulative number of positive and negative severe acute respiratory syndrome coronavirus 2 (SARS-CoV-2) cases and the number of positive and negative cases by date in the screening clinic of our university hospital.

\begin{tabular}{|c|c|c|c|c|c|c|}
\hline $\begin{array}{c}\text { Date } \\
\text { (YYYYMMDD) }\end{array}$ & $\begin{array}{c}\text { Number of Tested } \\
\text { Cases }\end{array}$ & $\begin{array}{c}\text { Cumulative Number } \\
\text { of Negative Cases }\end{array}$ & $\begin{array}{c}\text { Cumulative Number } \\
\text { of Positive Cases }\end{array}$ & Positive Rate & $\begin{array}{l}\text { Number of Negative } \\
\text { Cases Per Day }\end{array}$ & $\begin{array}{c}\text { Number of Positive } \\
\text { Cases Per Day }\end{array}$ \\
\hline 20200127 & 0 & 0 & 0 & - & 0 & 0 \\
\hline 20200128 & 0 & 0 & 0 & - & 0 & 0 \\
\hline 20200129 & 1 & 1 & 0 & 0 & 1 & 0 \\
\hline 20200130 & 1 & 2 & 0 & 0 & 1 & 0 \\
\hline 20200131 & 0 & 2 & 0 & - & 0 & 0 \\
\hline 20200201 & 1 & 3 & 0 & 0 & 1 & 0 \\
\hline 20200202 & 0 & 3 & 0 & - & 0 & 0 \\
\hline 20200203 & 0 & 3 & 0 & - & 0 & 0 \\
\hline 20200204 & 0 & 3 & 0 & - & 0 & 0 \\
\hline 20200205 & 0 & 3 & 0 & - & 0 & 0 \\
\hline 20200206 & 0 & 3 & 0 & - & 0 & 0 \\
\hline 20200207 & 0 & 3 & 0 & - & 0 & 0 \\
\hline 20200208 & 1 & 4 & 0 & 0 & 1 & 0 \\
\hline 20200209 & 0 & 4 & 0 & - & 0 & 0 \\
\hline 20200210 & 3 & 7 & 0 & 0 & 3 & 0 \\
\hline 20200211 & 2 & 9 & 0 & 0 & 2 & 0 \\
\hline 20200212 & 1 & 10 & 0 & 0 & 1 & 0 \\
\hline 20200213 & 0 & 10 & 0 & - & 0 & 0 \\
\hline 20200214 & 1 & 11 & 0 & 0 & 1 & 0 \\
\hline 20200215 & 0 & 11 & 0 & - & 0 & 0 \\
\hline 20200216 & 0 & 11 & 0 & - & 0 & 0 \\
\hline 20200217 & 1 & 12 & 0 & 0 & 1 & 0 \\
\hline 20200218 & 4 & 15 & 1 & 25.00 & 3 & 1 \\
\hline 20200219 & 11 & 26 & 1 & 0 & 11 & 0 \\
\hline 20200220 & 17 & 37 & 7 & 35.29 & 11 & 6 \\
\hline 20200221 & 23 & 57 & 10 & 13.04 & 20 & 3 \\
\hline 20200222 & 39 & 90 & 16 & 15.38 & 33 & 6 \\
\hline 20200223 & 50 & 135 & 21 & 10.00 & 45 & 5 \\
\hline 20200224 & 48 & 177 & 27 & 12.50 & 42 & 6 \\
\hline 20200225 & 56 & 228 & 32 & 8.93 & 51 & 5 \\
\hline 20200226 & 129 & 347 & 42 & 7.75 & 119 & 10 \\
\hline 20200227 & 277 & 605 & 61 & 6.86 & 258 & 19 \\
\hline 20200228 & 362 & 945 & 83 & 6.08 & 340 & 22 \\
\hline
\end{tabular}


Table 1. Cont.

\begin{tabular}{|c|c|c|c|c|c|c|}
\hline $\begin{array}{c}\text { Date } \\
\text { (YYYYMMDD) }\end{array}$ & $\begin{array}{c}\text { Number of Tested } \\
\text { Cases }\end{array}$ & $\begin{array}{c}\text { Cumulative Number } \\
\text { of Negative Cases }\end{array}$ & $\begin{array}{l}\text { Cumulative Number } \\
\text { of Positive Cases }\end{array}$ & Positive Rate & $\begin{array}{l}\text { Number of Negative } \\
\text { Cases Per Day }\end{array}$ & $\begin{array}{l}\text { Number of Positive } \\
\text { Cases Per Day }\end{array}$ \\
\hline 20200229 & 332 & 1256 & 104 & 6.33 & 311 & 21 \\
\hline 20200301 & 359 & 1603 & 116 & 3.34 & 347 & 12 \\
\hline 20200302 & 444 & 2019 & 144 & 6.31 & 416 & 28 \\
\hline 20200303 & 385 & 2390 & 158 & 3.64 & 371 & 14 \\
\hline 20200304 & 355 & 2728 & 175 & 4.79 & 338 & 17 \\
\hline 20200305 & 355 & 3075 & 183 & 2.25 & 347 & 8 \\
\hline 20200306 & 238 & 3307 & 189 & 2.52 & 232 & 6 \\
\hline 20200307 & 176 & 3482 & 190 & 0.57 & 175 & 1 \\
\hline 20200308 & 120 & 3599 & 193 & 2.50 & 117 & 3 \\
\hline 20200309 & 255 & 3848 & 199 & 2.35 & 249 & 6 \\
\hline 20200310 & 177 & 4020 & 204 & 2.82 & 172 & 5 \\
\hline 20200311 & 198 & 4216 & 206 & 1.01 & 196 & 2 \\
\hline 20200312 & 174 & 4387 & 209 & 1.72 & 171 & 3 \\
\hline 20200313 & 149 & 4536 & 209 & 0 & 149 & 0 \\
\hline 20200314 & 55 & 4590 & 210 & 1.82 & 54 & 1 \\
\hline 20200315 & 50 & 4639 & 211 & 2.00 & 49 & 1 \\
\hline 20200316 & 111 & 4747 & 214 & 2.70 & 108 & 3 \\
\hline 20200317 & 85 & 4832 & 214 & 0 & 85 & 0 \\
\hline 20200318 & 98 & 4929 & 215 & 1.02 & 97 & 1 \\
\hline 20200319 & 113 & 5042 & 215 & 0 & 113 & 0 \\
\hline 20200320 & 52 & 5093 & 216 & 1.92 & 51 & 1 \\
\hline 20200321 & 33 & 5126 & 216 & 0 & 33 & 0 \\
\hline 20200322 & 93 & 5219 & 216 & 0 & 93 & 0 \\
\hline 20200323 & 100 & 5319 & 216 & 0 & 100 & 0 \\
\hline 20200324 & 93 & 5412 & 216 & 0 & 93 & 0 \\
\hline 20200325 & 73 & 5484 & 217 & 1.37 & 72 & 1 \\
\hline 20200326 & 84 & 5568 & 217 & 0 & 84 & 0 \\
\hline 20200327 & 155 & 5723 & 217 & 0 & 155 & 0 \\
\hline 20200328 & 51 & 5774 & 217 & 0 & 51 & 0 \\
\hline 20200329 & 29 & 5803 & 217 & 0 & 29 & 0 \\
\hline 20200330 & 87 & 5890 & 217 & 0 & 87 & 0 \\
\hline 20200331 & 104 & 5994 & 217 & 0 & 104 & 0 \\
\hline
\end{tabular}


In total, 6211 individuals underwent SARS-CoV-2 testing. Overall, 5151 (82.93\%) and 1060 (17.07\%) individuals provided specimens through DT and conventional collection systems, respectively. Further, 217 individuals tested positive for SARS-CoV-2 (positive rate: 3.50\%). Of the 6211 individuals, 3368 were symptomatic or had a history of contact with COVID-19 patients, and 142 of them tested positive for SARS-CoV-2 (positive rate: $4.22 \%$ ). Further, 2843 patients were asymptomatic and had no history of contact with COVID-19 patients, and 75 of them tested positive for SARS-CoV-2 (positive rate: $2.64 \%$ ) (Table 2). A large number of individuals aged 20 to 59 years, $>1000$ per age range, underwent SARS-CoV-2 testing, and the positive rate was 1.5-4.6\% (Table 3). In contrast, a relatively small number of individuals aged 60 to 89 years underwent SARS-CoV-2 testing, but the positive rate was relatively high (6.8-14.0\%). Of the 211 patients with positive test results, 89 (41.01\%) were men, and 128 (58.99\%) were women.

Table 2. The number of positive SARS-CoV-2 cases and positive rate according to the presence of symptoms and history of contact with coronavirus disease 2019 (COVID-19) patients.

\begin{tabular}{cccc}
\hline $\begin{array}{c}\text { Classification of COVID-19 } \\
\text { Patients According to Symptom } \\
\text { or History of Contact }\end{array}$ & $\begin{array}{c}\text { Number of Tested } \\
\text { Individuals }(\boldsymbol{n})\end{array}$ & $\begin{array}{c}\text { Number of Positive } \\
\text { Cases }(\boldsymbol{n})\end{array}$ & $\begin{array}{c}\text { Positive Rate } \\
(\mathbf{\%})\end{array}$ \\
\hline $\begin{array}{c}\text { Symptomatic or having a history of } \\
\text { contact with COVID-19 patients } \\
\text { Asymptomatic and having } \\
\text { no history of contact with } \\
\text { COVID-19 patients }\end{array}$ & 3368 & 142 & 4.22 \\
\hline
\end{tabular}

Table 3. The number of tested individuals, number of SARS-CoV-2-negative and -positive cases, and positive rates according to age ranges.

\begin{tabular}{ccccc}
\hline Age (Years) & $\begin{array}{c}\text { Number of Tested } \\
\text { Individuals }(\boldsymbol{n})\end{array}$ & $\begin{array}{c}\text { Number of Negative } \\
\text { Cases }(\boldsymbol{n})\end{array}$ & $\begin{array}{c}\text { Number of Positive } \\
\text { Cases }(\boldsymbol{n})\end{array}$ & $\begin{array}{c}\text { Positive Rate } \\
(\%)\end{array}$ \\
\hline$<10$ & 103 & 101 & 2 & 1.94 \\
$10 \mathrm{~s}$ & 142 & 139 & 3 & 2.11 \\
$20 \mathrm{~s}$ & 1217 & 1185 & 32 & 2.63 \\
$30 \mathrm{~s}$ & 1333 & 1313 & 20 & 1.50 \\
$40 \mathrm{~s}$ & 1299 & 1271 & 28 & 2.16 \\
$50 \mathrm{~s}$ & 1219 & 1164 & 55 & 4.51 \\
$60 \mathrm{~s}$ & 628 & 585 & 43 & 6.85 \\
$70 \mathrm{~s}$ & 193 & 166 & 27 & 13.99 \\
$80 \mathrm{~s}$ & 68 & 61 & 7 & 10.29 \\
$90 \mathrm{~s}$ & 9 & 9 & 0 & 0 \\
\hline
\end{tabular}

During the 14-day period (26 February 2020 to 10 March 2020), when DT and conventional systems were administered simultaneously, 3964 individuals underwent SARS-CoV-2 testing. According to the screening system, the rates of positive test results were $4.11 \%$ (DT system) and 5.69\% (conventional system). Of the 3384 individuals who used the DT system, 1812 (53.55\%) were symptomatic or had a history of contact with COVID-19 patients, while 1572 (46.45\%) were asymptomatic and had no history of contact with COVID-19 patients. Of the 580 individuals who used the conventional system, $332(57.24 \%)$ were symptomatic or had a history of contact with COVID-19 patients, while $248(42.76 \%)$ were asymptomatic and had no history of contact with COVID-19 patients (Table 4).

Table 4. The number of SARS-CoV-2-positive cases and positive rates based on the presence of symptoms and history of contact with COVID-19 patients according to the screening system.

\begin{tabular}{cccc}
\hline $\begin{array}{c}\text { Classification of COVID-19 Patients } \\
\text { According to Symptom or } \\
\text { History of Contact }\end{array}$ & Drive-Through System & Conventional System & Total \\
\hline $\begin{array}{c}\text { Symptomatic or having a history of } \\
\text { contact with COVID-19 patients }\end{array}$ & $1812(53.55 \%)$ & $332(57.24 \%)$ & $2144(54.09 \%)$ \\
$\begin{array}{c}\text { Asymptomatic and having no history of } \\
\text { contact with COVID-19 patients }\end{array}$ & $1572(46.45 \%)$ & $248(42.76 \%)$ & $1820(45.91 \%)$ \\
$\quad$ Positive for COVID-19 & $139(4.11 \%)$ & $33(5.69 \%)$ & $172(4.34 \%)$ \\
\hline
\end{tabular}




\section{Discussion}

For managing pandemic diseases, such as COVID-19, not only accuracy but also the efficiency of diagnoses, such as the time taken to establish a diagnosis and the number of tests performed, are of utmost importance. Since SARS-CoV-2 is highly transmissible, rapid diagnosis and sufficient testing capacity are essential for the management of COVID-19 [1-3]. In our study, two specimen collection systems - conventional and DT-were examined. The DT system was approximately 9 times more efficient at collecting specimens for testing. These results suggested that the DT system was more efficient and useful for effective COVID-19 management. We believed that the wide-scale use of DT systems could significantly contribute to the efficient diagnosis of COVID-19.

In this study, positive results were seen in around $2.64 \%$ of asymptomatic individuals. These results supported that lack of symptoms should not be an indication of exclusion for SARS-CoV-2 testing. In other words, testing might be necessary during pandemics, even when if the patient is asymptomatic or has a history of contact with infected individuals $[5,6]$.

In our study, we found that older individuals (aged $\geq 60$ years) had a lesser tendency to undergo screening than younger individuals (aged $\leq 50$ years). The positive test result rates were higher among individuals in their $60 \mathrm{~s}-80 \mathrm{~s}$ than among those in the lower age groups. Older individuals had a lesser tendency to undergo the COVID-19 test than younger individuals. This could be due to the fact that many older individuals are physically weak or cannot drive. To develop appropriate strategies for managing COVID-19, the government and clinicians must focus more on the older population, especially individuals aged $\geq 60$ years.

During the study period, the number of tests and the cumulative number of positive patients began to increase exponentially from 20 February 2020. The main source of this rapid propagation was determined to be mass infection among a religious group (Shincheonji) in Daegu during a Sunday gathering. In this religious gathering, thousands of people were sitting close to each other in a confined space within the facility, having conversations, praying, and singing songs. The government had ordered screening tests for all the individuals in this group. Of them, $62 \%$ (>2000 cases) tested positive for SARS-CoV-2. Moreover, throughout South Korea, mass infections occurring in churches, hospitals, nursing homes, and call centers were largely responsible for the increase in the number of COVID-19 cases. Therefore, during the COVID-19 outbreak, group meetings should be restrained, and measures should be implemented in facilities where many people are gathered to actively prevent further COVID-19 cases as SARS-CoV-2 has a high transmission rate.

As of 11 April 2020, the total positive test result rate for SARS-CoV-2 in South Korea was 2.05\% (http://ncov.mohw.go.kr/en/). This value was lower than the positive test result rate of our study (3.49\%). This might be due to the fact that our hospital is located in Daegu, which was the epicenter of the COVID-19 outbreak in South Korea; as of 11 April 2020, around 65\% of COVID-19 patients in South Korea were Daegu residents.

\section{Conclusions}

In this study, we reviewed screening clinic data for SARS-CoV-2 in a single university hospital during the COVID-19 outbreak in Daegu, South Korea. The results of the analysis showed that the DT screening system was highly efficient. The DT system was a useful option for rapid and safe testing. Furthermore, our results showed a high positive test result rate in asymptomatic patients. Therefore, expanding screening tests to asymptomatic individuals might be necessary. Larger cohort studies analyzing data from multiple hospitals are warranted for acquiring more accurate information regarding SARS-CoV-2 and COVID-19.

Author Contributions: Data curation, W.-S.S. and J.H.; Writing-original draft, M.C.C. and D.P. All authors have read and agreed to the published version of the manuscript.

Funding: The present study was supported by a National Research Foundation of Korea grant funded by the Korean government, grant number NRF-2019M3E5D1A02068106. 
Conflicts of Interest: The authors declare no conflict of interest.

\section{References}

1. Chang, M.C.; Park, D. How should rehabilitative departments of hospitals prepare for coronavirus disease 2019? Am. J. Phys. Med. Rehabil. 2020, 99, 475-476. [CrossRef] [PubMed]

2. Kucharski, A.J.; Russell, T.W.; Diamond, C.; Liu, Y.; Edmunds, J.; Funk, S.; Eggo, R.M.; Centre for Mathematical Modelling of Infectious Diseases COVID-19 working group. Early dynamics of transmission and control of COVID-19: A mathematical modelling study. Lancet. Infect Dis. 2020, 20, P553-P558. [CrossRef]

3. Cascella, M.; Rajnik, M.; Cuomo, A.; Dulebohn, S.C.; Napoli, R.D. Features, Evaluation and Treatment Coronavirus (COVID-19); StatPearls publishing: Petersburg, FL, USA, 2020.

4. Kwon, K.T.; Ko, J.H.; Shin, H.; Sung, M.; Kim, J.Y. Drive-Through Screening Center for COVID-19: A Safe and Efficient Screening System against Massive Community Outbreak. J. Korean Med. Sci. 2020, 35, e123. [CrossRef] [PubMed]

5. Danis, K.; Epaulard, O.; Bénet, T.; Gaymard, A.; Campoy, S.; Bothelo-Nevers, E.; Bouscambert-Duchamp, M.; Spaccaferri, G.; Ader, F.; Mailles, A.; et al. Cluster of coronavirus disease 2019 (Covid-19) in the French Alps, 2020. Clin. Infect Dis. 2020, ciaa424. [CrossRef] [PubMed]

6. He, G.; Sun, W.; Fang, P.; Huang, J.; Gamber, M.; Cai, J.; Wu, J. The clinical feature of silent infections of novel coronavirus infection (COVID-19) in Wenzhou. J. Med. Virol. 2020. [CrossRef] [PubMed]

(C) 2020 by the authors. Licensee MDPI, Basel, Switzerland. This article is an open access article distributed under the terms and conditions of the Creative Commons Attribution (CC BY) license (http://creativecommons.org/licenses/by/4.0/). 